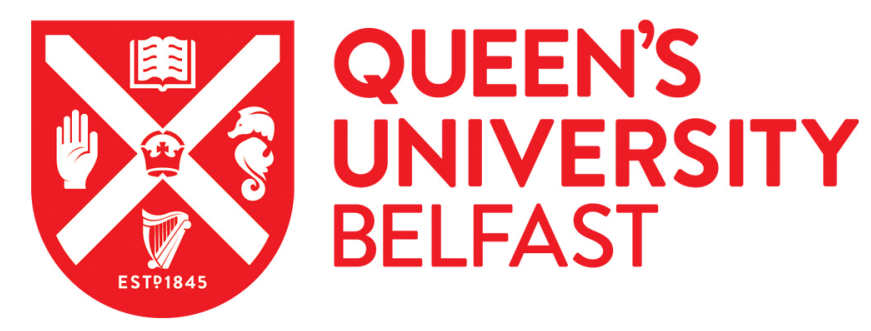

\title{
A timing-specific memory distortion effect in young children
}

McCormack, T., Brown, G., Smith, M. D., \& Brock, J. (2004). A timing-specific memory distortion effect in young children. Journal of Experimental Child Psychology, 87(1), 33-56. https://doi.org/10.1016/j.jecp.2003.10.001

Published in:

Journal of Experimental Child Psychology

Document Version:

Publisher's PDF, also known as Version of record

Queen's University Belfast - Research Portal:

Link to publication record in Queen's University Belfast Research Portal

\section{General rights}

Copyright for the publications made accessible via the Queen's University Belfast Research Portal is retained by the author(s) and / or other copyright owners and it is a condition of accessing these publications that users recognise and abide by the legal requirements associated with these rights.

Take down policy

The Research Portal is Queen's institutional repository that provides access to Queen's research output. Every effort has been made to ensure that content in the Research Portal does not infringe any person's rights, or applicable UK laws. If you discover content in the Research Portal that you believe breaches copyright or violates any law, please contact openaccess@qub.ac.uk. 


\title{
A timing-specific memory distortion effect in young children
}

\section{Teresa McCormack, ${ }^{*}$ Gordon D.A. Brown, Mark C. Smith, and Jon Brock}

University of Warwick, $U K$

Received 3 January 2003; revised 3 October 2003

\begin{abstract}
It has been suggested that there are systematic distortions in children's memory for temporal durations, such that children's memory is not just less accurate than that of adults but qualitatively different. Experiment 1 replicated the memory distortion effect by demonstrating developmental change in the tendency to confuse a reference duration with one that is shorter rather than longer than it. When the long-term memory demands of the task were reduced by providing reminders of the reference duration on every trial, there were no such qualitative changes in error patterns (Experiment 2). Further evidence for developmental changes in memory distortion was found in the temporal generalization task of Experiment 3, in which stimuli were spaced logarithmically rather than linearly. In Experiment 4, a similar distortion pattern was absent in a task in which children made judgments about the pitch rather than the duration of stimuli, suggesting the effect may be specific to time estimation.

(c) 2003 Elsevier Inc. All rights reserved.
\end{abstract}

Keywords: Time estimation; Time perception; Memory; Temporal generalization

\section{Introduction}

The development of temporal processing seems likely to underpin growth in a variety of cognitive and linguistic abilities. Developmental improvements in time

\footnotetext{
* Corresponding author. Present address: School of Psychology, Queen's University, David Keir Building, Malone Road, Belfast, Northern Ireland, BT9 5BP, UK. Fax: +44-2980-664144.

E-mail address: T.McCormack@qub.ac.uk (T. McCormack). 
estimation over childhood have been documented consistently in a large number of studies, using a wide variety of tasks (e.g., Arlin, 1986; Droit-Volet, 1999; Friedman, 1977; Pouthas \& Jacquet, 1987; for review, see Block, Zakay, \& Hancock, 1999).

That temporal abilities should improve with development is unsurprising. However, recent research has suggested that young children's memory for temporal durations is not just less accurate than that of older children and adults, but is instead qualitatively different. More specifically, it has been proposed that there is a developmental change in the extent to which durations are remembered as being shorter than they really are (Droit-Volet, Clement, \& Wearden, 2001; McCormack, Brown, Maylor, Darby, \& Green, 1999). Such a claim is of general theoretical interest for at least two reasons. First, the observation of a qualitative change rather than mere quantitative change requires specific explanation. Second, the possibility that there is memory distortion is specific to the temporal domain points to the possibility of developmental dissociations between development of memory for temporal and nontemporal quantities. This research addresses two key questions: Is the observed memory distortion specific to temporal memory? And is the effect best conceived of as memorial or perceptual in nature?

Recent attempts to examine the development of time estimation have been carried out within the framework of Scalar Expectancy Theory (Droit-Volet, 2002; DroitVolet \& Wearden, 2001; McCormack et al., 1999), the most widely applied theory of human and animal timing (Gibbon, 1977; Gibbon, Church, \& Meck, 1984; for review, see Wearden, 2001). Such studies have used duration identification tasks, in which participants are pre-exposed to one or more reference durations at the start of the task. At test, participants are required to judge the similarity of test durations to the reference duration(s) they encountered earlier.

According to Scalar Expectancy Theory, three types of processes are involved in such tasks. First, internal clock processes time the presented durations and generate representations of them that are passed to working memory. Second, long-term memory processes are responsible for storing and maintaining representations of the pre-exposed reference durations. Third, responses at test are based on the outcome of decision processes that compare representations of the reference durations with just-presented test durations. These processes are typically captured mathematically in scalar timing models (e.g., McCormack et al., 1999; Wearden, 1991, 1999; Wearden, Wearden, \& Rabbitt, 1997). A key advantage of carrying out developmental studies of timing within the Scalar Expectancy Theory framework is that such formal models enable developmental changes to be pinpointed in particular components of the processing stages. Specifically, developmental differences can be interpreted in terms of changes in one or more of the parameters specified in such models.

For present purposes, we highlight two important parameters. These parameters correspond to two different ways in which time estimation abilities may develop. On the one hand, there could be developmental changes in the amount of noise or variability associated with one or more of the processing stages. A developmental decrease in the amount of noise would lead to increases in overall levels of performance on duration identification tasks, but would not usually be associated with striking developmental changes in the patterns of errors that are produced on 
such tasks (at least according to typical scalar timing models). Alternatively, there could be qualitative developmental changes that are specific to the long-term memory processes recruited in the task. Scalar timing models assume that memory distortion effects can occur in certain populations (Droit-Volet et al., 2001; Elvevåg et al., 2003b; Lejeune, Ferrara, Soffie, Bronchart, \& Wearden, 1998; Malapani, Deweer, \& Gibbon, 2002; McCormack et al., 1999; McCormack, Brown, Maylor, Richardson, \& Darby, 2002; Meck, 1996) or under certain conditions (Meck, 1996; Meck \& Church, 1987). The idea is that durations can be systematically misrepresented in memory as too short or as too long. Developmental changes in distortion would lead not only to less accurate overall levels of performance, but also to characteristic patterns of errors in participants of different ages.

The McCormack et al. (1999) study of the development of duration identification employed a temporal generalization task to examine this issue. In the temporal generalization task, participants are presented with a single reference duration in an initial pre-exposure phase, and they then judge whether test durations are of the same length as the reference duration. Data from this task yield temporal generalization gradients, which are plots of the number of positive responses given to each test duration (such as those plotted in Fig. 1). Typically, these peak at the reference duration, and decline with distance from it. In studies of adults' performance on this task, such gradients are typically skewed to the right (right asymmetry) when the stimuli are spaced linearly, with more erroneous positive responses to stimuli longer than the reference duration than to those shorter but of equal distance away (Wearden, 1992; Wearden, Denovan, Fakhri, \& Haworth, 1997; Wearden, Wearden, et al., 1997). Thus, in adults, the confusability of durations would appear to be a function of the ratio between them, rather than the absolute distance. However, in the McCormack et al. study, 5-year-olds made more erroneous responses to stimuli shorter than rather than longer than the reference duration; that is, their gradients showed left asymmetry rather than the more usual right asymmetry. This tendency declined developmentally, with 10-year-olds producing symmetrical gradients. Thus, McCormack et al. found qualitative developmental differences in addition to quantitative ones. Such qualitative differences can be particularly helpful in identifying the specific aspects of cognitive processes that change developmentally.

In modeling their data, McCormack et al. (1999) assumed that noise or variability in performance decreased over childhood. This assumption captured the overall decline in numbers of errors. However, to capture the distinctive pattern of errors made by children, it was also necessary to assume that there was a developmental decline in the extent to which memory representations of the reference duration were distorted. Specifically, it was assumed that young children tend to remember the reference duration as being shorter than it really was, and thus make more erroneous identifications of short test stimuli with the standard. Varying both these parameters in a simple mathematical model of performance provided a good fit to the data from child and adult groups.

The idea that young children's temporal judgments are not simply less accurate than those of adults but also qualitatively different is one that has historically been the focus of much research on the development of timing. In the Piagetian 
framework, psychologists have been concerned with establishing whether children's duration judgments are susceptible to influence by nontemporal task features in the pre-operational period (Levin, 1982; Piaget, 1969). Another strand of research has explored whether young children systematically overestimate or underestimate temporal intervals due to a failure to accurately employ strategies such as counting (Levin \& Wilkening, 1989; Pouthas, 1993) or due to differences in attentional or physiological processes (e.g., Goldstone \& Goldfarb, 1966; Hicks, Allen, \& Mayo, 1984). However, as Block et al. (1999) discuss in their comprehensive meta-analytic review of this research, the claim that children overestimate or underestimate durations can be meaningfully interpreted only within the context of the particular type of timing task that is being employed. On the basis of their meta-analysis of studies that have used different types of timing tasks, Block et al. conclude that young children tend to make shorter reproductions of presented durations and make overestimations in verbal estimation tasks. Although simple theoretical interpretations of this pattern of findings are difficult, Block et al. interpret these findings in terms of changes in attentional processes and changes in the translation of subjective units of time to objective units of measurement (such as seconds or minutes).

Because the temporal generalization task involves making a duration judgment relative to a previously presented standard, distinctive patterns of errors can also be interpreted as overestimation or underestimation (i.e., overestimation or underestimation of the test interval relative to a remembered standard). The pattern of performance observed in young children by McCormack et al. (1999) can be described as overestimation, in that test durations that are shorter than the standard are erroneously judged to be of the same length as it. However, unlike in the verbal estimation tasks reviewed by Block et al. (1999), young children's pattern of errors in the temporal generalization task cannot be explained in terms of difficulties with objective units of time, because the judgments made on this task do not involve such units. The fact that McCormack et al. could successfully model their findings in terms of the distortion in long-term memory of the representation of the standard suggests that, in some circumstances, overestimation of durations by young children may be a result of changes in long-term memory processes. However, two issues remain. First, an explanation of the findings of McCormack et al. in terms of the perceptual processes, rather than the long-term memory processes, involved in timing needs to be ruled out. Second, the generality of the memory effect needs to be established. Block et al. (1999) discuss the possibility that a general developmental change in memory, such as a change in rate of forgetting, may lead to overestimation or underestimation of durations. However, the memory distortion process described by McCormack et al. is one that has been posited specifically by Scalar Expectancy Theory models of timing, and it is not, at least at present, assumed to be a general memory effect that might extend to other perceptual dimensions.

As they stand, the McCormack et al. findings suggest that there may be interesting developmental changes in long-term memory that may be specific to memory for durations. Existing research provides only mixed support for this claim. The main aim of this research was to establish whether this suggestion is correct. In another developmental study of timing carried out within the Scalar Expectancy Theory 
framework, Droit-Volet et al. (2001) examined the performance of a wide age range of children (their youngest group comprised 3-year-olds) on a duration identification task. Their task had a structure similar to that of McCormack et al., although DroitVolet et al. used much longer temporal durations. They also found qualitative changes in error patterns, and, like McCormack et al. (1999), assumed in modeling their data that there was a decreasing tendency with age to misremember a reference duration as shorter than it really was. In a more recent paper, however, Droit-Volet (2002) argued that it is not in fact necessary to assume such memory distortion to capture developmental changes in performance on temporal generalization tasks, and she obtained statistically good fits to her data without varying the memory distortion parameter in a scalar timing model.

Droit-Volet's (2002) more recent findings raise the issue of whether there are alternative explanations of the developmental changes in the asymmetry of temporal generalisation gradients found by McCormack et al. (1999). One such alternative explanation of the right asymmetry found in young children's gradients could be in terms of perceptual discrimination abilities. The basic finding of McCormack et al. is that this group gave an unusually large number of positive responses to the stimulus slightly shorter than the standard or reference duration and relatively few positive responses to stimuli slightly longer than the standard (the opposite of the pattern of errors in adults). Perhaps the specific short durations used in the task were not sufficiently different to be reliably perceptually discriminated from the standard by young children, even if there was no long-term memory component to the task. In other words, perhaps erroneous positive responses to short stimuli were not due to failure to remember the standard correctly but due to perceptual discrimination processes in young children that were relatively inaccurate for the specific range of short durations used in the task. We label this explanation the perceptual discrimination account of right asymmetry, to contrast it with an account in terms of a timing-specific memory distortion process. ${ }^{1}$

The aim of the current work was to reexamine the nature of young children's errors on a temporal generalization task, and to explore whether the memory distortion account is correct. The first experiment was an attempt to replicate the findings of McCormack et al. (1999) in a slightly older group of children (6-year-olds), because McCormack et al. found that a number of 5-year-olds had to be excluded from the study due to a failure to participate properly in the task. In the second experiment, the long-term memory load of the task was considerably reduced by providing reminders of the standard or reference duration on every trial. We were interested in whether qualitative changes with development in error patterns would be less evident when there was a low memory load, as a memory distortion account would predict. The last two experiments examine the generalizability of the developmental change in error patterns found by McCormack et al. (1999): Does it extend to a temporal generalization task involving a very differently spaced set of

\footnotetext{
${ }^{1}$ We are grateful to Nelson Cowan for suggesting this alternative explanation of our findings.
} 
durations (Experiment 3)? And does it extend to a stimulus dimension other than duration (Experiment 4)?

\section{Experiment 1}

\section{Method}

\section{Participants}

Nineteen 6-year-olds, twenty-eight 10-year-olds, and twenty adults participated in the study. The children were recruited from a primary school local to the University of Warwick and were tested individually in their school. The adults were undergraduates who participated voluntarily and received no payment for their participation.

\section{Materials}

The experimental stimuli were presented using an Apple Macintosh computer and controlled by a program written in the Psyscope software package. The auditory stimuli were $500-\mathrm{Hz}$ tones of different durations produced by the computer's speakers, and the visual displays were presented on a colored screen. Participants responded to stimuli by pointing to parts of the visual displays.

\section{Procedure}

Stimuli were presented in five blocks, with each block consisting of a series of eight trials, making a total of 40 experimental trials. The standard (reference) stimulus duration was $500 \mathrm{~ms}$, and the nonstandard durations were 125, 250, 375, 625, 750 , and $875 \mathrm{~ms}$. Each series of eight stimulus presentations contained one example of each of the nonstandard stimuli and two examples of the standard duration. Feedback was provided visually, and the delay between feedback and the next auditory event was of a variable length of time.

All participants were tested individually by one experimenter. Participants were told that they would hear some sounds and have to judge their length. The first phase of the introduction to the experiment was an initial exposure to the standard or reference stimulus. In this phase, a colored photograph of an owl appeared in the center of the screen. Participants were told that the owl's name was Barney, and that he always made a sound of the same length. They were instructed to listen carefully to how long the sound was. They then heard Barney's 500-ms sound five times, while the photograph of the owl remained in the center of the screen.

In the second phase of the introduction, participants were told that other owls, which were Barney's friends, made sounds of different lengths, some shorter and some longer than Barney's sound. A display appeared on the screen of two pictures side by side: One was the picture of Barney, and the other was the same picture of Barney, but with a large red cross through the picture. A nonstandard duration of 750-ms was played, and the experimenter explained that this was not Barney's sound because it was too long, and pointed to the crossed-out Barney. The 500-ms standard duration was then played, and the experimenter reminded the participant that this 
was Barney's sound, and pointed to the picture of Barney. Lastly, a 250-ms nonstandard duration was played, with the experimenter explaining that this was not Barney's sound as it was too short, and pointing to the crossed-out Barney.

A practice trial followed this demonstration, in which participants heard one example each of the four nonstandard durations that they had not yet encountered $(125,375,625$, and $875 \mathrm{~ms})$, plus another example of the standard duration. For each trial, a picture of Barney alongside a picture of a crossed-out Barney was displayed on the screen (an identical photograph overlaid with a large red cross), and participants responded by pointing to one of the pictures. After each response, participants were told whether they were correct.

Following the practice trial, participants were told that they would hear more sounds, and would have to judge again whether they were or were not Barney's sound. The last phase of the introduction was a second exposure to the standard duration: Barney's sound was played five more times, with the photograph of Barney again displayed in the center of the screen. The first experimental trial began immediately after these reminders. After the participant's response on each trial, a message was displayed on the bottom the screen that read either "That was Barney's sound" or "That was not Barney's sound." The experimenter also read this message aloud for child participants. Between trials, a visual prompt "Ready?" appeared on the screen, and participants were given the opportunity to have a short break if this were necessary.

\section{Results and discussion}

Fig. 1 shows the generalization gradients for each of the three age groups. It can be seen from the figure that there appears to be a developmental improvement in overall performance, as indicated by the group differences in the steepness of gradients. There also appears to be a developmental change in the symmetry of the gradient. The 6-year-old group make more errors to the stimulus immediately adjacent to but shorter than the $500-\mathrm{ms}$ reference stimulus $(375 \mathrm{~ms})$ than to the stimulus immediately adjacent to but longer than the $500-\mathrm{ms}$ reference stimulus $(625 \mathrm{~ms})$, the pattern we describe as left asymmetry. However, in the adult group this pattern is reversed, with this group showing the right asymmetry that is typical of temporal generalization gradients found in other studies of adults using similar stimulus sets. We analyze the results of all the experiments in this article in two complementary ways. First, conventional statistics are used to assess group differences and possible asymmetries in the generalization gradient. However, such analyses can be misleading when examining and interpreting symmetry in generalization gradients, in particular because separate underlying processes that might lead to asymmetries in opposite directions may interact in unpredictable ways. We therefore also interpreted the results of each experiment within a simple mathematical modeling framework as described subsequently.

A repeated-measures ANOVA was carried out on the proportion of positive responses to each stimulus, with a between-subject factor of group and a within-subject factor of duration. The main effect of group was significant, $F(2,64)=4.90, p<.01$, as were the main effect of duration, $F(6,384)=98.78, p<.01$, and the interaction 


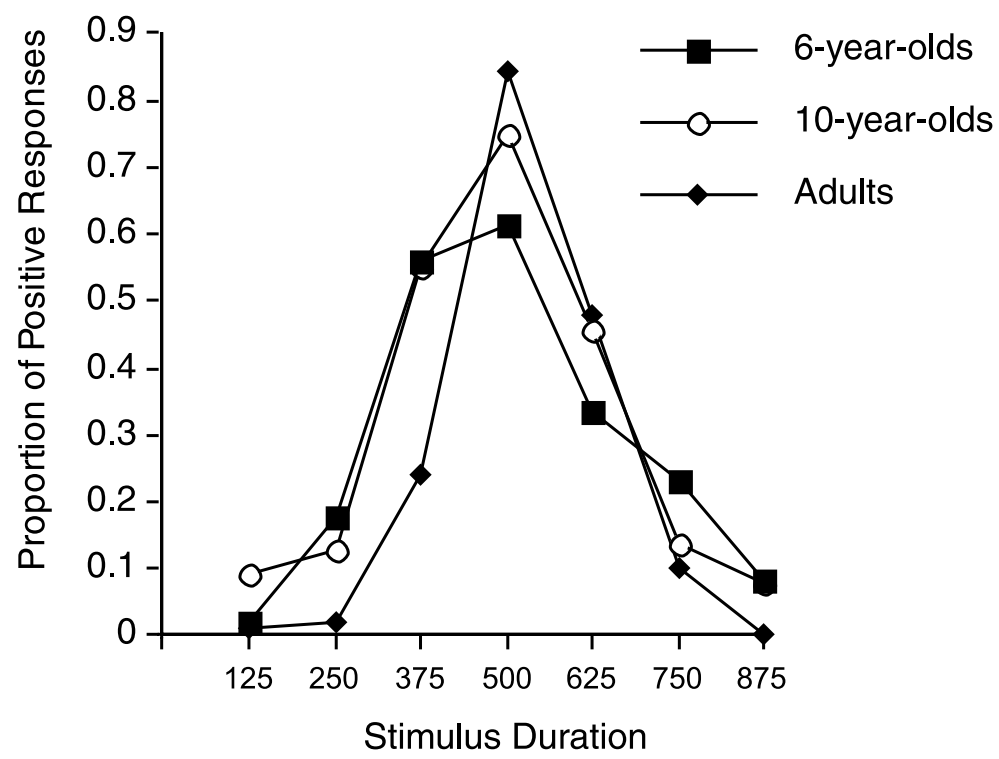

Fig. 1. Proportion of positive responses as a function of stimulus duration and age group in Experiment 1.

between group and duration, $F(12,384)=4.34, p<.01$. Post hoc analyses showed that the effect of group was significant for all durations apart from the 125-, 625-, and 750-ms stimuli (a significance level of $p<.05$ was set for these and subsequent analyses).

The symmetry of the gradients was examined by comparing the proportion of positive responses given to the 375-ms stimulus with that given to $625-\mathrm{ms}$ responses. The adult group showed significant right asymmetry, giving more positive responses to the 625 -ms stimulus, $t(19)=2.94, p<.01$, whereas the 6-year-old group showed significant left asymmetry, producing more positive responses to the $375-\mathrm{ms}$ stimulus, $t(18)=2.16, p<.05$. The 10 -year-old group did not show significant asymmetry of either type, $t(27)=0.97, p=.34$.

We also addressed these findings using a simple mathematical model. A variety of modeling frameworks have been applied to temporal generalization data; here we adopted the simplest possible approach in an attempt to gain understanding of the distortion effects and their developmental changes, even at the cost of possibly failing to account for every nuance of the data as could be done with a more complex model with a larger number of free parameters. The probability $P\left(Y \mid S_{i}\right)$, of responding "yes" to a given stimulus $S_{i}$ was given by

$$
P\left(Y \mid S_{i}\right)=b \cdot \eta_{i, s t},
$$

where $b$ is a scaling parameter and $\eta_{i, s t}$ is the similarity between the memory representation of the standard and the psychological representation of $S_{i}$. Standard practice (e.g., Nosofsky, 1986; Shepard, 1957) assumes that psychological similarity is an exponentially reducing function of psychological distance, but that a Gaussian 
function may provide the best fit when stimuli are perceptually confusable (see Ennis, 1988; Nosofsky, 1988; Shepard, 1988). A Gaussian similarity-distance function was used in the current model. We also assumed that temporal durations were represented internally on a logarithmic scale. (We note that alternative formulations, in terms, for example, of ratio-based decision rules, are also extant in the literature and are sometimes but not always equivalent to the formulation proposed here.) Thus, $R_{i}=\log \left(S_{i}\right)$, where $R_{i}$ is the internal representation of $S_{i}$. The internal representation of the standard duration, $R_{s t}$, is assumed to be a distorted version of the veridical representation of the standard, which would be $\log \left(S_{s t}\right)$. More specifically, $R_{s t}=\log \left(S_{s t}\right)+D$, where $D$ is the amount of distortion. Negative values of $D$ would result if the standard duration was remembered as shorter than it actually was.

As noted previously, similarity of any two items is a reducing Gaussian function of their separation in psychological space. Thus, if $c$ is the parameter governing the rate at which similarity decreases with distance,

$$
\eta_{i, m}=e^{-c\left|R_{i}-R_{s t}\right|^{2}} \text {. }
$$

In psychological terms, this simple model instantiates two basic assumptions. The first assumption is that the psychological similarity of any two temporal durations is related to the ratio of the shorter to the longer, subject also to perceptual noise. The second assumption is that the probability of responding "yes" to any given temporal duration in a temporal generalization task is directly proportional to the psychological similarity between the test duration and the remembered standard duration.

The key question to be answered by the model is whether a memory distortion parameter is consistently estimated as positive or negative across participants. We therefore fitted the model to the generalization curve of individual participants in each age group separately (minimizing sum-squared deviation between model and $\left.\mathrm{data}^{2}\right)$. This procedure enables statistical examination of the best-fitting parameter values. In particular, this procedure enables examination of whether estimates of the distortion parameter differ significantly from zero. If there is no systematic distortion in the data, then for any individual participant a better fit may be obtained by including either a small positive or small negative parameter (because parameter addition will almost invariably lead to improved fit). However, if the sign of the distortion parameter is consistent across participants (i.e., significantly different from zero), then it is reasonable to conclude that systematic distortion is present in the data. In some cases, modeling of the individual participant data revealed that no sensible model could be constructed due to deviant performance by an individual (e.g., when an individual responded "yes" on very few occasions throughout the

\footnotetext{
${ }^{2}$ Maximum likelihoods can be calculated directly from the response probabilities in this and subsequent experiments, but because of the high weight given to low observed response probabilities the resulting fits sometimes did less well at capturing the key effects relating to memory distortion. Rather than include additional parameters into the model to accommodate guessing or random responding, we report maximum likelihood parameters and test statistical significance by minimizing summed squared error. However, we carried out the model fitting and comparison both ways, and a qualitatively identical pattern of results was obtained in all cases.
} 
experiment). Data from such participants (two of the 10-year-olds in the present experiment) were not modeled. The resulting best-fit parameter values (averaged over participants) and mean and median $R^{2}$ values for the fit of the model to individual participants' data (with distortion included) are given in Table 1. All parameters varied in a meaningful way across age group. The $c$ parameter increased with age, as would be expected (see, e.g., McCormack et al., 2002). The distortion parameter, $D$, was negative for both 6-year-olds and 10-year-olds, consistent with the suggestion that the children were remembering the standard as shorter than its true value. The estimated distortion value for the adults was smaller in magnitude and opposite in direction, consistent with the suggestion that little or no distortion is involved in adult performance. The right asymmetry that is observed in the adult data is assumed in the model to be a straightforward consequence of representing durations internally on a logarithmic scale (see Wearden, 1992, for an alternative explanation in terms of the decision rule). The scaling parameter $b$ increased systematically with age. The main theoretical interest concerns the individual estimates of the distortion parameter $D$. Distortion was negative and significantly different from zero for the 6-year-olds, $t(18)=3.2, p<.01$, and for the 10 -year-olds, $t(25)=2.7, p<.05$, but was nonsignificant for the adults, $t(19)=1.04, p>.10$.

In summary, the findings of this study closely replicate those of McCormack et al. (1999), in that both quantitative and qualitative developmental changes in errors were found. There was an overall significant improvement in performance, as indicated by the developmental change in the steepness of the generalization gradient. Furthermore, there was a change in error patterns, with the groups of children producing more errors to the stimulus adjacent to but shorter than the reference duration, and the adult group showing the reverse pattern of errors. Specifically, the findings suggest that younger children remember durations as shorter than they

Table 1

Parameter values and model fits

\begin{tabular}{llrrrrr}
\hline & Age group & \multicolumn{2}{l}{ Parameter } & \multirow{2}{*}{$R^{2}$ mean } & $R^{2}$ median \\
\cline { 3 - 5 } & & $c$ (SE) & $D(\mathrm{SE})$ & $b(\mathrm{SE})$ & & \\
\hline Experiment 1 & 6-year-olds & $7.7(1.4)$ & $-.15(.05)$ & $.76(.05)$ & .798 & .818 \\
& 10-year-olds & $10.0(1.1)$ & $-.07(.03)$ & $.87(.03)$ & .884 & .930 \\
& Adults & $20.6(3.0)$ & $.02(.02)$ & $.93(.02)$ & .946 & .975 \\
Experiment 2 & 6-year-olds & $7.6(1.5)$ & $.13(.09)$ & $.76(.05)$ & .862 & .919 \\
& 10-year-olds & $21.2(4.5)$ & $-.03(.03)$ & $.88(.04)$ & .945 & .879 \\
& Adults & $33.7(8.7)$ & $.02(.01)$ & $.97(.01)$ & .983 & .993 \\
Experiment 3 3 & 6-year-olds & $7.1(1.3)$ & $-.10(.06)$ & $.72(.06)$ & .839 & .911 \\
& 10-year-olds & $9.3(1.6)$ & $-.11(.03)$ & $.88(.04)$ & .932 & .979 \\
& Adults & $14.1(1.5)$ & $.02(.02)$ & $.95(.02)$ & .969 & .995 \\
Experiment 4 4 & 6-year-olds & $80.7(22.9)$ & $.007(.02)$ & $.75(.05)$ & .809 & .899 \\
& 10-year-olds & $88.3(11.6)$ & $.004(.009)$ & $.84(.03)$ & .865 & .921 \\
& Adults & $133(18.5)$ & $.025(.009)$ & $.92(.03)$ & .874 & .947 \\
\hline
\end{tabular}


actually were. These conclusions were supported by parameter estimates derived from fitting a simple mathematical model to the data (see also McCormack et al., 1999, 2002).

\section{Experiment 2}

Scalar Expectancy Theory models of performance on the standard temporal generalization task assume that a representation of the reference duration is held in long-term memory and subsequently retrieved and compared with durations presented at test. The distortion process is assumed to occur in the transfer (Meck, 1996) or maintenance of reference durations in long-term memory. This assumption leads to the prediction that such distortion effects will be seen only on tasks that require use of long-term memory representations, and that similar patterns of errors will be produced by all age groups in tasks that do not involve use of such representations. In Experiment 2, reminders of the reference duration were provided on every trial, allowing performance to be based primarily on working memory rather than long-term memory representations. Thus, each trial consisted of participants judging whether two stimuli were of the same duration, although the first stimulus in every pair was always the reference duration. The memory distortion account predicts that all age groups should show similar error patterns in these circumstances, because distortion effects may be absent (or at least reduced), given the reduced reliance on long-term memory representations.

This experimental manipulation also allows us to test the perceptual discrimination account of left asymmetry described above. According to this alternative account, children's positive responses to the stimulus shorter than the standard may be due to children's inability to discriminate between this specific short stimulus and the (accurately remembered) standard when they are held and compared in working memory, because of relatively inaccurate perceptual discrimination of short stimuli. If the perceptual discrimination account is correct, right asymmetry should still be observed even if reminders of the standard are provided on every trial, because the asymmetry is not thought to be due to a problem with long-term memory for the standard.

\section{Method}

\section{Participants}

Seventeen 6-year-olds, eighteen 10-year-olds, and twenty-two adults participated in the study. The children were recruited from a primary school local to the University of Warwick, and were tested individually in their schools. The adults were primarily undergraduates at the University of Warwick, who participated voluntarily and received no payment. None of the participants had taken part in Experiment 1.

\section{Materials}

The materials used were identical to those in Experiment 1. 


\section{Procedure}

The procedure differed from that used in Experiment 1 in only two respects. First, the standard stimulus was introduced to participants as "Marvin the mouse's sound" (rather than that of Barney the owl), and the pictures used on the computer display were a photograph of a mouse and a crossed-out mouse. Second, participants were reminded of Marvin's sound before they made each judgement. On each experimental trial, the photograph of Marvin appeared on the top of the screen. After $1000 \mathrm{ms,}$ the 500-ms standard sound was played. After a further $1000 \mathrm{~ms}$, the screen went blank for $1000 \mathrm{~ms}$, and then a photograph of Marvin alongside a crossed-out photograph of Marvin appeared at the bottom of the screen. After a further $1000 \mathrm{~ms}$, the test stimulus was presented. Thus, each experimental trial consisted of a presentation of Marvin's sound, an interstimulus interval of $3000 \mathrm{~ms}$, and finally presentation of the test stimulus. Participants were asked to judge whether the second stimulus in each pair was Marvin's sound.

\section{Results and discussion}

Fig. 2 shows the generalization gradients for all three groups of participants. All three groups have generalization gradients that show some degree of right asymmetry (i.e., typically, more errors are made to stimuli longer rather than shorter than the standard). This can be contrasted with the findings of Experiment 1, in which the youngest group showed significant left asymmetry.

A repeated-measures ANOVA was carried out on the proportion of positive responses given to each stimulus, with a between-subject factor of group and a

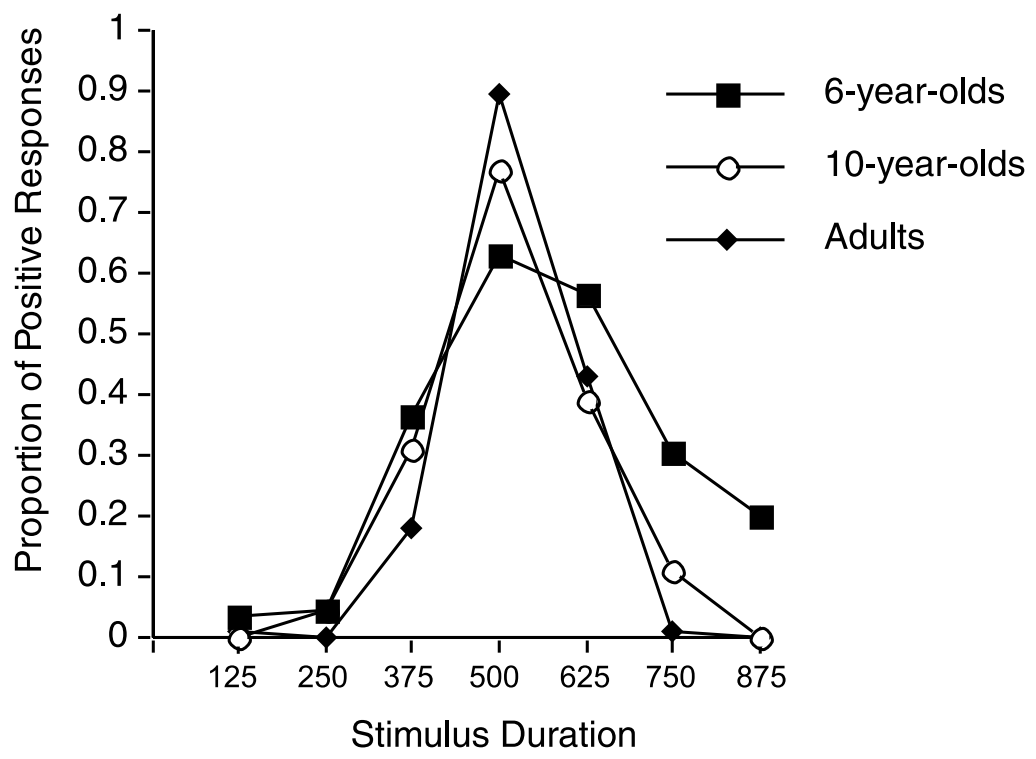

Fig. 2. Proportion of positive responses as a function of stimulus duration and age group in Experiment 2. 
within-subject factor of duration. There was a significant main effect of group, $F(2,53)=5.92, p<.01$, a significant effect of duration, $F(6,318)=152.51, p<.01$, and a significant interaction between group and duration, $F(12,318)=6.21$, $p<.01$. Post hoc analyses revealed that the group effect was significant for the 500-, 750 -, and 875-ms durations only, with the only significant differences being between the 6-year-olds and the older two groups.

To examine asymmetry, the proportions of positive responses given to each of the pair of durations adjacent to the reference duration (i.e., 375 and $625 \mathrm{~ms}$ ) were compared using $t$ tests. In the 6-year-old group and in the adult group, significantly more errors were made to the longer stimulus in the pair, $t(16)=2.75, p<.02$, and $t(20)=4.02, p<.01$ respectively. Although the 10 -year-old group tended to make more errors to the longer stimulus, this difference was not significant, $t(17)=0.86$, $p=.40$.

We applied the same model as was used for Experiment 1 to the data and the bestfitting parameter estimates are shown in Table 1. As for Experiment 1, estimates for both the $c$ and $b$ parameters increased in an orderly way with age. More importantly, in no age group did the parameter estimates indicate a significant tendency to misremember the standard duration as too short. Indeed, if anything, the youngest children behaved more like the adults in both Experiments 1 and 2 in that a small amount of positive distortion was possibly evident. Analysis of the individual parameter estimates, after exclusion of one participant from each age group for whom a meaningful model could not be constructed, found no significant distortion for the 6 -year-olds, $t(15)=1.6, p>.10$, the 10 -year olds, $t(16)=1.3, p>.10$, or the adults, $t(20)=1.5, p>.10$.

In this experiment, participants received a reminder of the reference duration on each trial, which allowed judgments to be made on the basis of working memory representations rather than primarily on long-term memory representations. In such circumstances, although there were still significant improvements in performance with age, the striking qualitative developmental differences in error patterns that had been found in Experiment 1 and by McCormack et al. (1999) were not present. Parameter estimates derived from the model led to converging conclusions, in that no negative distortion was evident in any of the groups. This suggests that the findings of the previous studies are indeed due to the demands on long-term memory of the tasks employed in those studies. These findings are evidence against what we have described as the perceptual discrimination account of right asymmetry. When the participant's task was simply to discriminate between two just-presented durations, no left asymmetry was observed, even in the youngest group. This suggests that young children do not have particular difficulties discriminating between the short stimulus durations used in the task.

\section{Experiment 3}

According to a memory distortion account, young children should make more errors to stimuli shorter than the reference stimulus, regardless of the spacing of the 
stimuli in the set. An alternative account remains to be excluded however. In magnitude estimation tasks using nontemporal stimuli (e.g., weights or light intensities), it is often found that the psychological mean of a set of stimuli is influenced by the spacing of the stimuli within the set (see e.g., Parducci, 1995, for a review). It is at least possible, therefore, that if participants' memory of the standard duration is in some way contaminated with the psychological mean of the stimulus set, distortion effects might be removed or reduced when the spacing of stimuli changes. To ensure the generalizability of the distortion-based account, we decided to replicate the basic effect using logarithmically based stimuli, as no previous experiment has examined developmental changes in temporal generalization using stimuli spaced in this way.

In Experiment 3, participants completed a temporal generalization task again, with the minimum and maximum test stimuli being identical to those in Experiments 1 and 2 (125 and $875 \mathrm{~ms}$ ), but with the intermediate five stimuli arranged so that they were evenly spaced on a logarithmic rather than linear scale. A memory distortion account again predicts left asymmetry in young children's generalization gradients, because remembering the reference duration as shorter than it actually is should result in a greater number of erroneous positive responses to stimuli shorter than the reference duration. Based on the findings of previous studies of temporal generalization using log-spaced stimuli in adults, the generalization gradients of the adult group in Experiment 3 are predicted to be approximately symmetrical (Wearden, Denovan, et al., 1997). Finally, it was predicted that model-based estimation of distortion parameters would lead to parameter values close to those obtained in Experiment 1.

\section{Method}

\section{Participants}

Twenty five 6-year-olds ( $M=6$ years, 2 months; 14 males and 11 females), twentyfive 10-year-olds ( $M=10$ years, 4 months; 10 males and 15 females), and twenty-five adults ( 9 males and 16 females) took part in the study. The adults were undergraduate students who took part in a 30-min testing session during which they completed the current task plus an unrelated visual discrimination task. They were paid $5 \mathrm{UK}$ pounds for their participation in the testing session.

\section{Materials}

The materials were identical to those used in Experiment 1, except for the durations of the test stimuli which were $125,173,239,331,457,633$, and $875 \mathrm{~ms}$, with $331 \mathrm{~ms}$ being the standard stimulus.

\section{Procedure}

The procedure was identical to that used in Experiment 1.

\section{Results and discussion}

The data for two of the 6-year-olds who produced similar numbers of positive responses for all stimulus durations were removed from the analyses, as it was judged 
that they were unlikely to have understood or followed task instructions. Fig. 3 shows the temporal generalization gradients for the remaining participants in each of the three groups. The generalization gradients are steeper in the older groups, indicating a developmental improvement in overall levels of performance. It can be seen from the figure that the generalization gradient of the adult group is approximately symmetrical. The gradient for the 6-year-old group showed partial evidence of left asymmetry in that "yes" responses were somewhat more likely for stimuli shorter than the standard than those longer than the standard. However, the left asymmetry appeared less clear than in the gradient of the 10-year-old group, who showed such an effect strongly.

A repeated-measures ANOVA with a between-subject factor of group and a within-subject factor of duration was used to examine the data. The main effect of group was not significant, $F(2,70)=1.29, p=.28$, indicating similar levels of overall number of positive responses across groups. The main effect of duration was significant, $F(6,420)=133.36, p<.01$, and there was also a significant interaction between group and duration, $F(12,420)=5.98, p<.01$. Post hoc analyses showed that age effects were significant at the 125-, 239-, 331-, and 633-ms durations. To examine the symmetry of the gradients, $t$ tests were used to compare the proportion of positive responses to the two stimuli immediately adjacent to the 500 -ms reference stimulus. There was no significant difference between these proportions in the 6-year-old group, $t(22)=0.43, p=.67$, or in the adult group, $t(24)=0.92, p=.37$, but the 10 year-old group made significantly more positive responses to the stimulus adjacent to but shorter than $500 \mathrm{~ms}, t(24)=3.57, p<.01$.

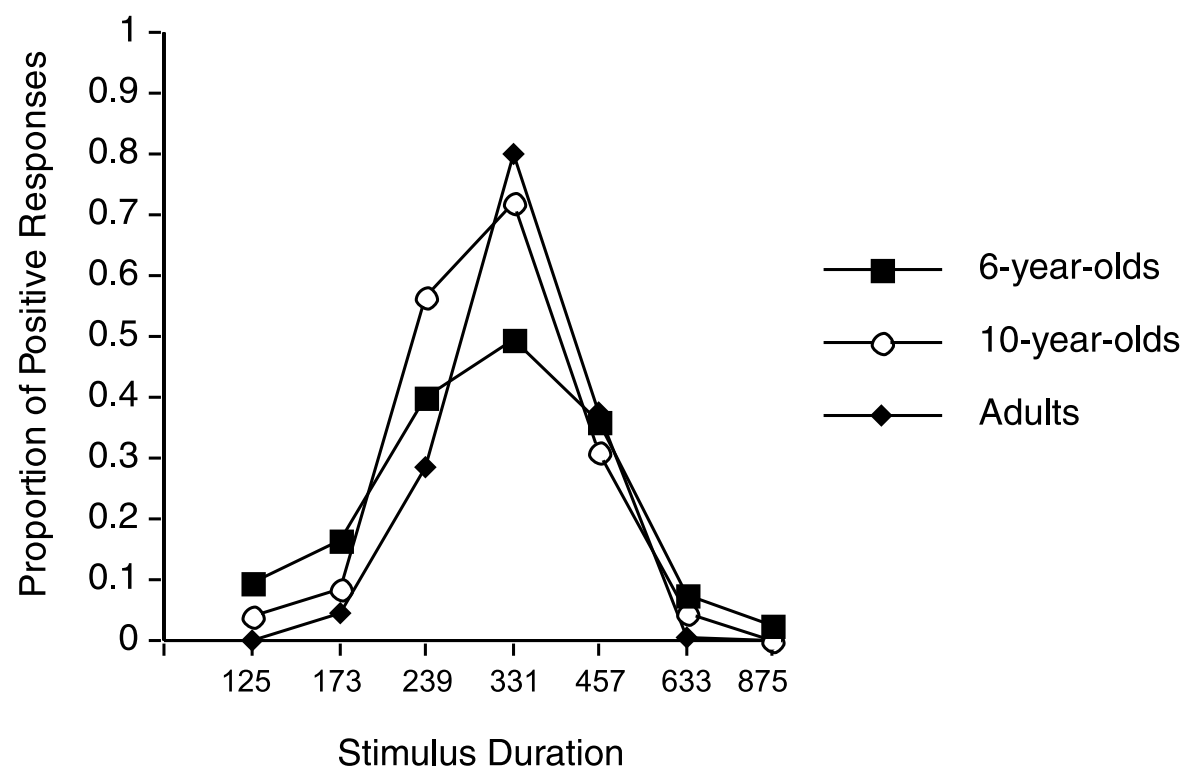

Fig. 3. Proportion of positive responses as a function of stimulus duration and age group in Experiment 3. 
The apparent absence of left asymmetry in the youngest group is difficult to interpret on the basis of the data alone, because of the relatively low level of performance in the youngest group, as compared with the equivalent group in Experiment 1. We therefore examined the findings using the same simple mathematical model as used previously.

The resulting parameter estimates are given in Table 1. As in the previous two experiments, estimates of the $c$ and the $b$ parameters increased with age. Of critical interest were the estimates of the distortion parameter. As in the model of the data from Experiment 1 , the mean individual distortion parameter estimates were negative for the two groups of children but small and positive for the adult group. Prior to modeling, data from two of the 6-year-olds were excluded as a meaningful model could not be constructed. Analysis of the individual parameter estimates just failed to find significant distortion for the 6-year-olds, $t(20)=1.8, p>.10$, but found a clear effect for the 10 -year-olds, $t(24)=3.4, p<.01$, whereas no effect was found for the adults, $t(24)$ $=0.8, p>.10$.

In summary, as in previous studies, there was a significant developmental improvement in overall performance on a temporal generalization task. The finding of symmetrical generalization gradients in adults when log-spaced stimulus sets are used is compatible with previous studies (Wearden, Denovan, et al., 1997), and is predicted by typical Scalar Expectancy Theory models of human timing. Model-based estimates of distortion were generally consistent with those obtained in Experiment 1, and consistent with the hypothesis that distortion effects do not depend on the spacing (linear versus logarithmic) of the stimuli.

\section{Experiment 4}

The findings of Experiments 1-3 are all compatible with the memory distortion account. In attributing developmental changes in the shape of the generalization gradient to a memory distortion effect, we are suggesting that the observed developmental differences are due to a process that may be unique to timing. The long-term memory distortion process has been posited specifically in the temporal domain by Scalar Expectancy Theory models of time estimation (Lejeune et al., 1998; Meck, 1983, 1996), and we are not aware of any similar claims regarding the memory for representations of other stimulus dimensions.

In a previous study of developmental decline in stimulus identification in old age, we found evidence for a memory distortion effect in a duration identification task, but not in a pitch identification task (McCormack et al., 2002). In that study, error patterns in the duration identification task were modeled by assuming that elderly adults remembered the reference durations as longer than they really were - in effect, the opposite type of distortion that appears to be evident in children's performance. The findings from the duration identification task of McCormack et al. were compatible with those of Lejeune et al. (1998) in their study of rats, who found evidence that aging rats seemed to remember a reference duration as longer than it really was. 
On the basis of the McCormack et al. (2002) findings of a duration-specific memory distortion effect in elderly adults, we might expect to find the developmental effect in children also to be specific to duration. This possibility was examined in Experiment 4 using a modified version of the generalization task, in which the dimension along which stimuli varied was pitch rather than duration. We predicted that there would be overall age-related changes in levels of performance, but that there would not be developmental changes in the nature of error patterns. Specifically, we predicted that the children's generalization gradients would not show the left asymmetry found in our previous experiments.

\section{Method}

\section{Participants}

Twenty five 6-year-olds ( $M=6$ years, 5 months; 13 males and 12 females), twentyfive 10-year-olds ( $M=10$ years, 3 months; 11 males and 14 females), and twenty-five adults (17 males and 8 females) took part in the study. The adults were undergraduate students who took part in a 20 -min testing session during which they completed the current task and an unrelated time estimation task. They were paid 4 UK pounds for their participation. Children were recruited from primary schools in Birmingham, $\mathrm{UK}$, and were tested individually in their schools.

\section{Materials}

The materials were identical to those used in Experiment 1, except for the stimulus set. The stimuli were 500-ms tones that varied in frequency; the standard stimulus was $900 \mathrm{~Hz}$ and the nonstandard stimuli were 600, 700, 800, 1000, 1100, and $1200 \mathrm{~Hz}$.

\section{Procedure}

The procedure was identical to that of Experiment 1, except that, during the training phase, nonstandard stimuli were described as "too high" or "too low" to be Barney's sound.

\section{Results and discussion}

Six 6-year-olds were excluded from the analysis because they produced similar numbers of positive responses to the extreme nonstandard stimuli as they did to the standard, suggesting that they did not understand the task. Fig. 4 shows the gradients for each of the three age groups on the pitch version of the generalization task. It can be seen from the figure that there is an overall improvement in level of performance with age, with steeper generalization gradients in the older groups. However, the general pattern of errors seems to be reasonably similar for all three age groups, with none of the groups showing the distinctive left asymmetry observed in our studies of temporal generalization (see Fig. 1). A two-way ANOVA on the proportion of positive responses to each stimulus, with a between-subject factor of group and a within-subject factor of pitch, confirmed these findings. There was a main effect of 


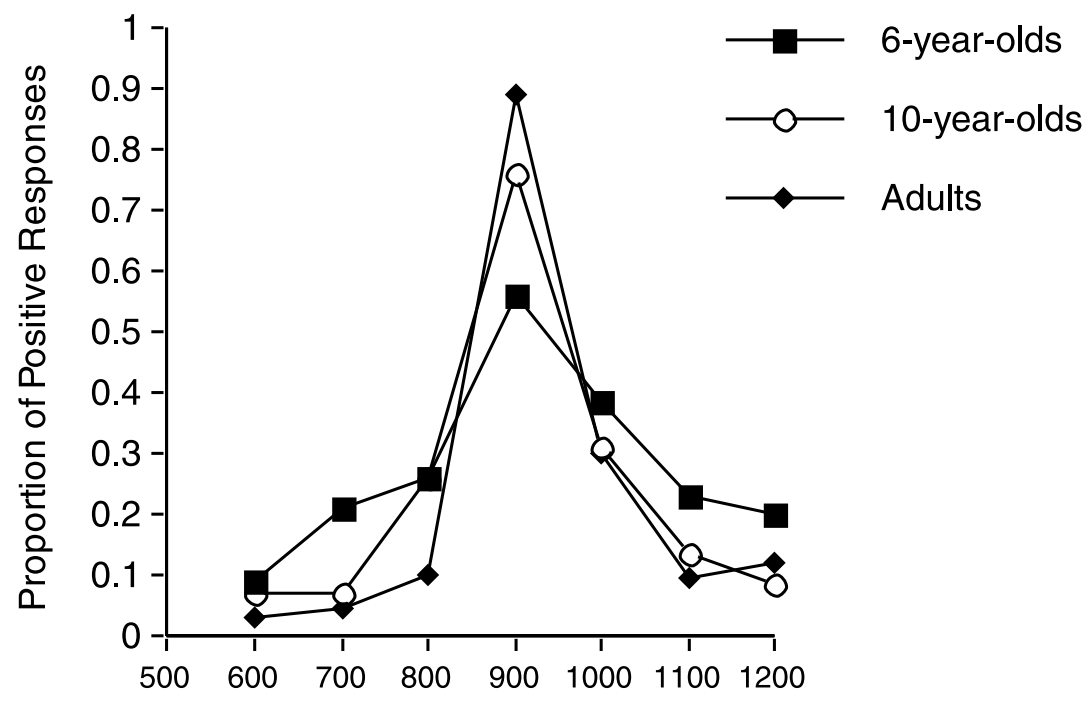

Pitch $(\mathrm{Hz})$

Fig. 4. Proportion of positive responses as a function of pitch and age group in Experiment 4.

pitch, $F(6,396)=108.45, \quad p<.001$, but no significant main effect of age, $F(2,66)=1.92, p=.16$, indicating participants of all age groups gave similar numbers of positive responses. The interaction between age and pitch was significant, $F(12,396)=5.38, p<.001$, and subsequent analyses showed that the effect of age was significant for the 700-, 800-, 900-, and $1100-\mathrm{Hz}$ stimuli. To examine asymmetry, the proportion of positive responses to the $800-\mathrm{Hz}$ stimulus was compared with the proportion of positive responses to the $1000-\mathrm{Hz}$ stimulus for each group. These proportions were significantly different for the adult group, $t(24)=3.27, p<.01$, but the difference failed to reach significance for the 6-year-old group, $t(18)=1.34$, $p=.20$, and the 10-year-old group, $t(24)=.62, p=.54$.

We examined the findings using the same simple mathematical model as used to examine temporal generalization in Experiments 1 through 3. Prior to modeling, data from four of the 6-year-olds, three of the 10-year-olds, and four of the adults were excluded as a meaningful model could not be constructed for the data from these participants. The parameter estimates are shown in Table 1. As in the previous experiments, estimates of the $c$ and the $b$ parameters increased systematically with age group. Of critical interest were the estimates of the distortion parameter. The mean individual distortion parameter estimates were small in magnitude and positive in sign for all groups. Analysis of the individual parameter estimates found that the estimated distortion parameter value did not differ from zero for the 6-year-olds, $t(14)=0.31, p>.10$, or for the 10 -year olds, $t(21)=0.41, p>.10$, but was significantly greater than zero for the adults, $t(20)=2.74, p<.05$. 


\section{General discussion}

The aim of this article was to examine further the claim made by McCormack et al. (1999) and Droit-Volet et al. (2001) that there are qualitative, in addition to quantitative, developmental changes in performance on duration identification tasks. Specifically, McCormack et al. suggested that younger children, unlike adults of undergraduate age, have distorted memories for brief durations: Such durations are remembered as being shorter than they actually were. This claim was made on the basis of successfully modeling data from a temporal generalization task by varying the memory distortion parameter in a simple model of timing similar to those typically employed in the Scalar Expectancy Theory framework. Experiment 1 closely replicated the findings of the previous study of McCormack et al. with the modeling of the data again being compatible with a distortion account. When the spacing of the stimuli in the set was altered (Experiment 3), so that the test durations were equally spaced psychologically (i.e., logarithmically rather than linearly spaced), qualitative developmental changes in error patterns were again found, and these patterns were successfully modeled by assuming distortion in children's long-term memory for durations.

The findings of Experiment 2 ruled out an alternative explanation of the left asymmetry found in young children's temporal generalization gradients. According to a perceptual discrimination account, errors to stimuli shorter than the standard are not due to a distorted memory for the standard, but are due instead to children's difficulties actually discriminating between a pair of relatively short stimuli (i.e., the short nonstandard test stimulus and an accurately remembered standard). In Experiment 2, the heavy demands of the generalization task on long-term or reference memory were reduced, with participants required on each test trial to discriminate between a justpresented pair of stimuli that could both be held in working memory. Performance still improved developmentally, but there were no striking qualitative changes in error patterns. These findings suggested that interesting developmental changes may indeed lie in the accurate recruitment of long-term memory representations of the pre-exposed reference duration, rather than merely in the discrimination of durations.

Perhaps the most important findings were those of Experiment 4, in which participants completed a pitch rather than a temporal generalization task. As in studies of temporal generalization, there were age-related improvements in overall levels of performance. However, the patterns of errors were similar for all age groups, and there was no evidence of distortion effects. Overall levels of performance for each age group were similar to those in Experiment 1, suggesting that the differences between the tasks in error patterns were not simply due to differential task difficulty. Although we have examined only one other perceptual dimension, the findings suggest that the developmental changes in memory distortion may be specific to long-term memory for durations (see also McCormack et al., 2002). This finding is important, because it suggests that changes in a general memory process, such as domain-general changes in rate of forgetting, cannot explain the findings of Experiment 1. Rather, the long-term memory process in question may be one that is specific to the representation and storage of duration information. 
The idea that representations of durations can be distorted in memory stems from neuropharmacological studies with animals (see Gibbon, Malapani, Dale, \& Gallistel, 1997; Meck, 1983, 1996). Based on such studies, Meck (1996) has suggested that transferring a representation of a duration from working memory to long-term or reference memory may involve a translation process that is prone to systematic error. Put simply, $n$ units of time in working memory may be translated as $n \cdot x$ units of time in long-term memory. If $x$ is less than 1, durations will be stored as shorter than they really were, whereas if $x$ is greater than 1, durations will be stored as longer than they really were. They found that the changes in the patterns of errors made by rats when administered drugs that altered levels of cholinergic activity were consistent with the idea that neuropharmacological manipulations affected a hypothesized timing-specific translation process in systematic ways.

Although the findings of Meck and colleagues have supported the idea that memory distortion can occur in timing tasks, it is not clear whether all distortion effects observed in humans have the same underlying basis. For example, there may be ways of explaining some distortion effects that do not appeal to the notion of erroneous translation processes: Representations of reference durations might become distorted not as a result of how they are encoded into long-term memory, but as a result of memory degradation that occurs during storage or through a faulty retrieval process (see Malapani et al., 2002). At present, further research is necessary investigating the details of the processes that lead to memory distortion, and indeed to establish whether effects seen in human populations share an underlying basis with those seen in animals.

Although the details of the processes underlying memory distortion may be unclear, the idea that timing-specific long-term memory processes have a distinctive effect on children's duration judgments is important in the light of previous cognitive explanations of the development of timing. The current tasks involve making comparative judgments about durations in the range of milliseconds. Strategies such as counting cannot be employed and the tasks also do not require familiarity with standard units used to measure time. Thus, many previous explanations of group differences in terms of higher-level cognitive developmental changes are unlikely to be appropriate (Block et al., 1999; Pouthas, 1993). However, an important issue raised by the current study is whether memory distortion effects that we have observed also occur in situations in which judgments about much longer durations are required. Arguably, the finding of Block et al. (from their meta-analysis of a number of studies) that young children tend to produce shorter reproductions of durations in the range of seconds (rather than milliseconds) could also be explained in terms of distorted memory for the to-be-reproduced duration. Block et al. suggest that young children make shorter reproductions simply because they are impatient and thus terminate their reproduction too soon. However, they point out that, alternatively, children's difficulty in waiting during empty time intervals may be a result of experiencing the duration as being longer than it actually is. The idea that children have distorted long-term memory representations of durations may be a way of giving substance to the idea that young children's experiences of time periods is distorted in some way. 
The final, broader, theoretical issue that we consider concerns the similarity of the processes used in duration identification and those used in perceptual identification. Historically, Scalar Expectancy Theory models of timing and duration identification have been developed largely independently of more general models of perceptual identification. However, in our own modeling work (McCormack et al., 1999, 2002), we have used a model that has been influenced by previous research on identification and categorization (e.g., Brown, Neath, \& Chater, 2002; Nosofsky, 1986). Furthermore, we have found that human temporal processing can be accounted for with the same models that have been used to model processing of other perceptual dimensions such as pitch (Brown, McCormack, Smith, \& Stewart, 2003). However, despite the formal similarities between models, there are also good reasons to believe that timing and duration identification may be served by mechanisms that are neuropsychologically dissociable from those employed in the processing and identification of other perceptual dimensions. First, clinical research has found impairments in certain populations that appear to be specific to duration identification (e.g., Elvevåg et al., 2003a; Nichelli, Alway, \& Grafman, 1996). Second, we have found that elderly adults' patterns of errors on a duration identification task are qualitatively different from those on a pitch identification task of equal difficulty (McCormack et al., 2002), a finding that is reminiscent of the current findings. A third relevant finding comes from Wearden's (Wearden, Parry, \& Stamp, 2002) recent work on "subjective shortening" (see also Spetch \& Wilkie, 1983), in which varying retention interval seems to have a distinctive effect on error patterns that is specific to duration judgments, and is not found when the judgments involve another perceptual dimension such as line length.

The current findings are compatible with the claim that duration identification involves mechanisms that are developmentally dissociable from those used in other types of perceptual identification. However, we note that our specific interpretation of our findings hinges on taking measures of asymmetry of generalization gradients as an index of memory distortion. Although we have tried to rule out at least one alternative interpretation of developmental change in the asymmetry of such gradients, it is clear from the literature on temporal generalization in humans and animals that interpreting the shape of generalization gradients is not straightforward. Studies with animals tend to yield symmetrical gradients (Church \& Gibbon, 1982), whereas studies with human adults tend to find gradients with right asymmetry (McCormack et al., 1999; Wearden, 1992; Wearden, Wearden, et al., 1997), with the humananimal difference sometimes accounted for in terms of differences in decision rules (Wearden, 1992; Wearden, Denovan, et al., 1997). The shape of adult temporal generalization gradients is also affected by whether feedback is provided (Wearden, Pilkington, \& Carter, 1999) and by task instructions that may affect decision rules (Wearden \& Grindrod, 2003).

Although, as far as we are aware, gradients with left asymmetry have not been reported in any studies of human adults, the possibility remains that the patterns of errors that we have found in young children may result from a factor or combination of factors that is yet to be identified, rather than from memory distortion. Indeed, we note that the findings of Droit-Volet's (2002) study, which used a similar 
range of stimuli, are less compatible with such an account (although there was some evidence of left asymmetry in her younger two groups of children). How can we reconcile the current findings with those of Droit-Volet (2002)? One possibility is that distortion effects are more evident when performance is relatively uncontaminated by random positive responses (see Droit-Volet, 2002), and performance is relatively accurate (as in the current study, especially in Experiment 1). Another possibility is that differences in procedural details and sample sizes are somehow responsible for the contrasting findings. Additional research on developmental changes in time estimation within the Scalar Expectancy Theory framework is necessary to resolve these issues.

Although such issues remain unresolved, the findings of the current study and the success of fitting the results using a simple mathematical model of timing provide further evidence that research on developmental changes in timing within the broad framework of Scalar Expectancy Theory is fruitful. An advantage of carrying out developmental research within this framework is that it allows comparison of children's performance on timing tasks with that of other groups, such as the elderly (McCormack et al., 1999; Wearden, Wearden, et al., 1997) and clinical groups (Elvevåg et al., 2003a, 2003b). Rather than simply comparing overall levels of performance across such groups, modeling the data allows us to examine whether parameters need to be varied in contrasting ways to capture different types of group differences. The findings of the current studies suggest that there may be developmental changes in a memory distortion process that lead young children to remember durations as being shorter than they really were, and that these developmental changes in memory distortion may be specific to duration identification.

\section{Acknowledgments}

This research was supported by grants from the BBSRC (88/S15050) and the ESRC (R000 23 9002) awarded to the first and second authors.

\section{References}

Arlin, M. (1986). The effects of quantity and depth of processing on children's time perception. Journal of Experimental Child Psychology, 42, 84-98.

Block, R. A., Zakay, D., \& Hancock, P. A. (1999). Developmental changes in human duration judgments: A meta-analytic review. Developmental Review, 19, 183-211.

Brown, G. D. A., McCormack, T., Smith, M. C., \& Stewart, N. (2003). Common mechanisms in bisection of identification of tones and times: A range-frequency analysis. Manuscript in preparation.

Brown, G. D. A., Neath, I., \& Chater, N. (2002). A ratio model of scale-invariant memory and identification. Manuscript submitted for publication.

Church, R. M., \& Gibbon, J. (1982). Temporal generalization. Journal of Experimental Psychology: Animal Behavioral Processes, 8, 165-186.

Droit-Volet, S. (1999). Time estimation in young children: Effects of response type and familiarity. Current Psychology of Cognition, 18, 27-44.

Droit-Volet, S. (2002). Scalar timing in temporal generalization in children with short and long stimulus durations. Quarterly Journal of Experimental Psychology, 55A, 1193-1209. 
Droit-Volet, S., Clement, A., \& Wearden, J. (2001). Temporal generalization in 3- to 8-year-old children. Journal of Experimental Child Psychology, 80, 271-288.

Droit-Volet, S., \& Wearden, J. (2001). Temporal bisection in children. Journal of Experimental Child Psychology, 80, 142-159.

Elvevåg, B., Brown, G. D. A., McCormack, T., Vousden, J. I., Weinberger, D. R., \& Goldberg, T. E. (2003a). Absolute identification of tone duration, line length and letter position: An experimental approach to working memory deficits in schizophrenia. Manuscript submitted for publication.

Elvevåg, B., McCormack, T., Gilbert, A., Brown, G. D. A., Weinberger, D. R., \& Goldberg, T. E. (2003b). Duration judgments in patients with schizophrenia. Psychological Medicine, 33, 1249-1261.

Ennis, D. M. (1988). Confusable and discriminable stimuli: Comment on Nosofsky (1986) and Shepard (1986). Journal of Experimental Psychology: General, 117, 408-411.

Friedman, E. R. (1977). Judgments of time intervals by young children. Perceptual and Motor Skills, 45, $715-720$.

Gibbon, J. (1977). Scalar expectancy theory and Weber's law in animal timing. Psychological Review, 84, $278-325$.

Gibbon, J., Church, R. M., \& Meck, W. H. (1984). Scalar timing in memory. In J. Gibbon \& L. G. Allan (Eds.), Timing and time perception (pp. 52-77). New York: New York Academy of Sciences.

Gibbon, J., Malapani, C., Dale, C. L., \& Gallistel, C. R. (1997). Towards a neurobiology of temporal cognition: Advances and challenges. Current Opinion in Neurobiology, 7, 170-184.

Goldstone, S., \& Goldfarb, J. L. (1966). The perception of time by children. In A. H. Kidd \& J. L. Rivoire (Eds.), Perceptual development in children (pp. 445-486). New York: International Universities Press.

Hicks, R. E., Allen, D. A., \& Mayo, J. P. (1984). A developmental study of temporal duration judgments. Journal of Genetic Psychology, 144, 31-38.

Lejeune, H., Ferrara, A., Soffie, M., Bronchart, M., \& Wearden, J. H. (1998). Peak performance in young adult and aged rats: Acquisition and adaptation to a changing temporal criterion. Quarterly Journal of Experimental Psychology, 51B, 193-227.

Levin, I. (1982). The nature and development of time concepts: The effect of interfering cues. In W. J. Friedman (Ed.), The psychology of time (pp. 47-85). New York: Academic Press.

Levin, I., \& Wilkening, F. (1989). Measuring time via counting: The development of children's conception of time as a quantifiable dimension. In I. Levin \& D. Zakay (Eds.), Time and human cognition: A life span perspective (pp. 119-144). Amsterdam: North Holland.

Malapani, C., Deweer, B., \& Gibbon, J. (2002). Separating storage from retrieval dysfunction of temporal memory in Parkinson's disease. Journal of Cognitive Neuroscience, 14, 311-322.

McCormack, T., Brown, G. D. A., Maylor, E. A., Darby, R. J., \& Green, D. (1999). Developmental changes in time estimation: Comparing childhood and old age. Developmental Psychology, 35, 1143 1155 .

McCormack, T., Brown, G. D. A., Maylor, E. A., Richardson, L. B. N., \& Darby, R. J. (2002). Effects of aging on absolute identification of duration. Psychology and Aging, 17, 363-378.

Meck, W. H. (1983). Selective adjustment of the speed of the internal clock and memory processes. Journal of Experimental Psychology: Animal Behavior Processes, 9, 171-201.

Meck, W. H. (1996). Neuropharmacology of timing and time perception. Cognitive Brain Research, 3, $227-242$.

Meck, W. H., \& Church, R. M. (1987). Cholinergic modulation of the content of temporal memory. Behavioral Neuroscience, 101, 465-475.

Nichelli, P., Alway, D., \& Grafman, J. (1996). Perceptual timing in cerebellar degeneration. Neuropsychologia, 34, 863-872.

Nosofsky, R. M. (1986). Attention, similarity, and the identification-categorization relationship. Journal of Experimental Psychology: General, 115, 39-57.

Nosofsky, R. M. (1988). On exemplar-based exemplar representations: Reply to Ennis (1988). Journal of Experimental Psychology: General, 117, 412-414.

Parducci, A. (1995). Happiness, pleasure, and judgment: The contextual theory and its applications. Mahwah, NJ: Erlbaum.

Piaget, J. (1969). The child's conception of time. New York: Basic Books. 
Pouthas, V. (1993). Ontongenesis of temporal learning in the child: Experimental evidence and perspectives. Psychologica Belgica, 33, 171-183.

Pouthas, V., \& Jacquet, A. Y. (1987). A developmental study of timing behavior in $4 \frac{1}{2}$ - and 7 -year-old children. Journal of Experimental Child Psychology, 43, 282-299.

Shepard, R. N. (1957). Stimulus and response generalization: A stochastic model relating generalization to distance in psychological space. Psychometrika, 22, 325-345.

Shepard, R. N. (1988). Time and distance in generalization and discrimination: Reply to Ennis (1988). Journal of Experimental Psychology: General, 117, 415-416.

Spetch, M. L., \& Wilkie, D. M. (1983). Subjective shortening: A model of pigeon's memory for event durations. Journal of Experimental Psychology: Animal Behavior Processes, 9, 14-30.

Wearden, J. H. (1991). Do humans possess an internal clock with scalar timing properties? Learning and Motivation, 22, 59-83.

Wearden, J. H. (1992). Temporal generalization in humans. Journal of Experimental Psychology: Animal Behavior Processes, 18, 134-144.

Wearden, J. H. (1999). Beyond the fields we know: Exploring and developing scalar timing theory. Behavioural Processes, 45, 3-21.

Wearden, J. (2001). Internal clocks and the representation of time. In C. Hoerl \& T. McCormack (Eds.), Time and memory: Issues in philosophy and psychology (pp. 37-58). Oxford: Oxford University Press.

Wearden, J. H., Denovan, L., Fakhri, M., \& Haworth, R. (1997). Scalar timing in temporal generalization in humans with longer stimulus durations. Journal of Experimental Psychology: Animal Behavior Processes, 23, 502-511.

Wearden, J. H., \& Grindrod, R. (2003). Manipulating decision processes in the human scalar timing system. Behavioural Processes, 61, 47-56.

Wearden, J. H., Parry, A., \& Stamp, L. (2002). Is subjective shortening in human memory unique to time representations? Quarterly Journal of Experimental Psychology, 55B, 1-25.

Wearden, J. H., Pilkington, R., \& Carter, E. (1999). "Subjective lengthening" during repeated testing of a simple temporal discrimination. Behavioural Processes, 46, 25-38.

Wearden, J. H., Wearden, A. J., \& Rabbitt, P. M. A. (1997). Age and IQ effects on stimulus and response timing. Journal of Experimental Psychology: Human Perception and Performance, 23, 962-979. 